\title{
Contribuição do uso de medicamentos para a admissão hospitalar
}

\author{
Patrícia de Carvalho Mastroianni", Fabiana Rossi Varallo, Marina Souza Barg, \\ Ana Regina Noto, José Carlos Fernandez Galduróz
}

\author{
Departamento de Fármacos e Medicamentos, Faculdade de Ciências Farmacêuticas, \\ Universidade Estadual Paulista "Júlio de Mesquita Filho"
}

\begin{abstract}
As Reações Adversas a Medicamentos (RAM) podem ser responsáveis por 2,4\% a 11,5\% das admissões hospitalares. O estudo objetivou conhecer o perfil demográfico dos pacientes internados por possíveis RAM, identificar os medicamentos e as queixas mais freqüentemente relacionadas e estimar a incidência de admissão hospitalar pelo uso de medicamentos. Durante um mês, os pacientes internados em um hospital geral foram entrevistados quanto ao uso de medicamentos antes da internação e as queixas que o levaram ao hospital; as informações foram analisadas, usando-se a base de dados MICROMEDEX ${ }^{\circledR}$ e outras bases oficiais. Observou-se que as admissões por uso de medicamentos ocorreram predominantemente em idosos [47,5\% (66/139)] e mulheres [62\% (87/139)]. Os medicamentos mais freqüentes foram: omeprazol (16), analgésicos (31), antihipertensivos (31), sinvastatina (7) e formoterol (6); e normalmente os sintomas associados foram do sistema digestório $(20,5 \%)$, circulatório $(20,2 \%)$, respiratório $(18,2 \%)$ e SNC (13,9\%). Estima-se que em 15,5\% (139/897) das internações, possivelmente, a razão foi o uso de medicamentos. Os dados sugerem medidas de prevenção, como o acompanhamento farmacoterapêutico dos pacientes no âmbito da assistência primária à saúde, principalmente aos idosos, aos portadores de doenças crônicas e aos polimedicados, além da orientação farmacêutica na compra e dispensação de medicamentos, principalmente os isentos de prescrição.
\end{abstract}

Unitermos: Medicamentos/uso racional. Medicamentos/reações adversas. Atenção farmacêutica. Acompanhamento farmacoterapêutico

According to the Word Health Organization, adverse drug reactions (ADR) are any harmful and non intentional answer which occurred in doses normally used in human beings. The ADR can be responsible for $2.4 \%$ to $11.5 \%$ of hospital admissions. Therefore, this study aimed at knowing the admitted patient's demographic profile due to possible ADR, identifying the most frequent drugs and complaints, and evaluating the incidence of hospital admission related to drug use. Patients who were 18 years old or more and were admitted during a period of one month to a medical clinical of a general hospital were interviewed for one month about drug use before being admitted, as well as regarding to the complaint which led them to hospital. These information were analyzed according to official data, like MICROMEDEX ${ }^{\circledR}$ and WHO criteria as well. It was observed that the admission due to drug use occurred in most part of the cases in elderly [47.5\% (66/139)] and women [62\% (87/139)]. The most frequent drugs used were: omeprazole (16), analgesics (31), antihypertensive (31), simvastatin (7) and formoterol fumarate (6), and the symptoms were normally associated to the digestive $(20.5 \%)$, circulatory $(20.2 \%)$, respiratory $(18.2 \%)$ and central nervous systems (13.9\%). It was estimated that $15.5 \%(139 / 897)$ of the hospital admission occurred possibly due to the drug use. The data found by present study suggests some strategies in order to prevent ADR in the context of primary health care services, such as monitoring drug therapy, manly for patients with chronic diseases, elderly and polimedicated people; and pharmaceutical care including dispensation and purchasing of the drugs, a lot of them dispensed over the counter (OTC).

Uniterms: Drugs/rational use. Drugs/adverse reactions. Pharmaceutical attention. Pharmacotherapeutic care.

\footnotetext{
*Correpondência: P. C. Mastroianni. Faculdade de Ciências Farmacêuticas, Universidade Estadual Paulista "Júlio Mesquita Filho", Rodovia: Araraquara - Jaú Km 1 - 14801-902 - Araraquara - SP, Brasil. E-mail: pmcastro@fcfar.unesp.br
} 


\section{INTRODUÇÃO}

De acordo com a Organização Mundial da Saúde, reações adversas a medicamentos (RAM) são definidas como sendo qualquer evento nocivo e não intencional que tenha ocorrido na vigência do uso de medicamento, em doses normalmente usadas em humanos, com finalidades terapêutica, profilática ou diagnóstica. Portanto, não se incluem entre as RAM as superdosagens (acidentais ou intencionais) e a ineficácia do medicamento para o tratamento proposto.

Estima-se que entre 2,4\% a $11,5 \%$ das admissões hospitalares estão relacionadas com as RAM (Green et al., 2000; Hallas et al., 1990; Howard et al., 2003; Junntti, 2002; Pirmohamed et al., 2004; Pfaffenbach et al., 2002; Scheneweiss et al., 2001; Zargarzadeh et al., 2007); contudo, dependendo da especialidade médica, enfermaria e o método de análise das RAM, a prevalência poderá variar de $0,2 \%$ a $40 \%$. Os fatores que favorecem a ocorrência das RAM e admissões hospitalares são: a idade, o número de medicamentos ingeridos, o gênero (Onder et al., 2002), a presença de comorbidades e doenças renais (Caamaño et al., 2005).

Estudos têm demonstrado que a prevalência é maior em idosos e mulheres (Caamaño et al., 2005; Mannesse a et al., 2000; Mannesse b et al., 2000; Pouyanne et al., 2000). Os idosos são quatro vezes mais hospitalizados por RAM do que os não idosos (Beijer et al., 2002). Outros fatores sóciodemográficos tais como, nível educacional(Pfaffenbach et al., 2002), morar sozinho, estado civil, o uso de álcool ou tabaco não demonstraram interferência na prevalência de internações por RAM (Caamaño et al., 2005).

Ao analisarem sistematicamente 68 estudos observacionais de RAM como causa de admissões hospitalares, Beijer et al. (2002) identificaram que as classes terapêuticas mais comumente relacionadas eram os cardiovasculares (38 estudos), antiinflamatórios não esteróides (AINE) e analgésicos (30 estudos), antidiabéticos (12 estudos), antineoplásicos (8 estudos), diuréticos (20 estudos), anticoagulantes (19 estudos) e corticóides (17 estudos).

As complicações mais freqüentes são sintomas do trato gastrintestinal (Caamaño et al., 2005; Mannesse a et al., 2000; Onder et al., 2002; Zargarzadeh et al., 2007), hemorragias (Mannesse b et al., 2000; Onder et al., 2002; Pouyanne et al., 2000); complicações metabólicas (Caamaño et al., 2005; Onder et al., 2002) e síncopes (Caamaño et al., 2005). Nos idosos os sintomas mais comuns são hepáticos, renais e cognitivos (Caamaño et al., 2005). Juntti-Patinen e Neuvonen (2002) observaram que em 5\% de todos os óbitos de um hospital universitário, certamente ou provavelmente, o uso de medicamento estava relacio- nado, o que corresponde a $0,05 \%$ de todas as admissões do hospital estudado.

O paciente hospitalizado por RAM pode ter o seu tempo de internação aumentado em 1,7 dias e a média do tempo de internação chega a 7,69 dias, conseqüentemente, há aumento nos custos totais com internação (Classen et al., 1997). No entanto, $67 \%$ a $75 \%$ dos casos poderiam ser prevenidos, porque são conhecidas as propriedades farmacológicas, o que demonstra que as RAM são negligenciadas (Howard et al., 2003; Onder et al., 2002) e subnotificadas.

No Brasil, a coordenação e planejamento das ações de farmacovigilância tiveram início com a criação da ANVISA em 1999 e, apenas em 2002, com a implantação da rede sentinela, iniciou-se a busca ativa de notificações no âmbito hospitalar. Conseqüentemente, são recentes e poucos os dados farmacoepidemiológicos de notificações de RAM em nosso país (Pfaffenbach et al., 2002).

Portanto, há necessidade de estudos nacionais para estimar as possíveis internações hospitalares relacionadas ao uso de medicamento, conhecer o perfil demográfico (gênero e faixa etária) dos pacientes possivelmente internados por RAM, bem como conhecer quais são os fármacos e as queixas mais freqüentemente relacionadas com internação hospitalar, a fim de propor medidas preventivas.

\section{MÉTODO}

Trata-se de um estudo farmacoepidemiológico, descritivo e observacional. As investigações epidemiológicas de cunho descritivo têm o objetivo de informar sobre a distribuição de um evento, na população, em termos quantitativos, identificar grupos de risco e sugerir explicações para as variações de freqüência (Pereira, 1995).

\section{Local}

O estudo foi realizado em hospital geral particular, com 132 leitos, no município de Araraquara, com a média de 750 internações/mês. Realizou-se a coleta nas clínicas médicas (88 leitos), por ser a porta de entrada para admissão hospitalar, pois o pronto atendimento tem características de ambulatório.

As clínicas do berçário, UTI adulto, UTI neonatal, pediatria, centro cirúrgico e a obstetrícia foram excluídas, porque os sujeitos da pesquisa são apenas os pacientes maiores de 18 anos, cuja razão de internação poderia ser o uso de medicamento antes da admissão hospitalar.

\section{Sujeitos}

Critérios de inclusão: pacientes maiores de 18 anos 
que aceitassem participar da pesquisa e assinassem o termo de consentimento livre e esclarecido.

Critérios de exclusão: pacientes com cirurgia préagendada, partos, cesáreas, transferidos de outro hospital ou de outra enfermaria, internação inferior a 24 horas, ou dados insuficientes.

\section{Período}

Os dados foram coletados diariamente na clínica médica durante o mês de maio de 2006.

\section{Formulário elaborado para a coleta e análise dos dados}

O formulário foi elaborado com a finalidade de orientar a entrevista e possibilitar a introdução das informações pesquisadas para posterior tabulação dos dados.

A Parte I continha informações de identificação: sexo (masculino ou feminino) e faixa etária (entre 18 a 40 anos; entre 41 a 60 anos e maiores de 60 anos); leito e dia de internação; e a pergunta se o paciente fez ou fazia uso de algum medicamento antes da internação, em caso positivo, quais eram e quais as queixas que o levaram ao hospital. As informações das queixas eram confirmadas com o prontuário médico.

A Parte II continha espaço para preencher as informações pesquisadas: os fármacos dos medicamentos informados pelos pacientes e sua classificação terapêutica (ATCAnatomical Therapeutic Chemical Code) (WHO, 2008); tipo de prescrição do medicamento (isento de prescrição, sob prescrição ou sob controle especial (Brasil, 1998)); essencialidade do medicamento (presente ou ausente na lista RENAME (Brasil, 2006)) e a classificação da queixa segundo o código internacional das doenças - CID-10 (Ministério da Saúde, 1998).

A relação de possível causalidade entre a queixa da internação e o uso do medicamento antes da admissão hospitalar de cada paciente foi estabelecida segundo os critérios da OMS para possiveis RAM (WHO, 1972). As bases oficiais consultadas sobre os fármacos foram o bulário da ANVISA (disponível em: http://bulario.bvs.br/) e MICROMEDEX® (em: www.portaldapesquisa.com.br; disponível gratuitamente para as universidades públicas e hospitais sentinela).

\section{Tabulação e análise dos dados}

Foi criado um banco de dados para organizar as informações contidas nos formulários em duas planilhas do aplicativo Excel. A primeira planinha continha as informações: faixa etária, sexo, uso ou não de medicamentos, identificação ou não de RAM pelo sujeito da pesquisa; e a segunda planilha continha as informações dos sujeitos que utilizaram medicamentos e sabiam quais eram: classe terapêutica dos fármacos, tipo de prescrição, essencialidade, e a classificação CID-10 do motivo da internação e identificação ou não de RAM.

A associação entre as categorias foi analisada utilizando o teste do qui-quadrado (disponível em: http:// faculty.vassar.edu/lowry/VassarStats.html)

\section{Aprovação do Comitê de Ética em Pesquisa}

O projeto de pesquisa foi aprovado pelo Comitê de Ética em Pesquisa da UNIFESP, sob protocolo ${ }^{\circ} 173 / 07$.

\section{RESULTADOS}

Durante o levantamento dos dados, 897 pacientes foram internados na clínica médica. Destes 562 não participaram da pesquisa por apresentarem os critérios de exclusão: 209 tinham cirurgias pré-agendadas; 145 não estavam em condições de participar da pesquisa e não foram entrevistados, respeitando o princípio ético de não maleficiência; 97 eram partos ou cesáreas; 70 eram menores de 18 anos, 25 não aceitaram participar do estudo e 16 eram transferidos de outras unidades do hospital.

Participaram da pesquisa 335 pacientes. Destes, 266 haviam ingerido pelo menos um medicamento, sendo que $52(20 \%)$ não sabiam ou não lembravam o nome de qualquer um dos medicamentos que haviam ingerido. Foram analisadas as informações de 214 pacientes que lembravam o nome de pelo menos um dos medicamentos que haviam ingerido antes da internação (Figura 1).

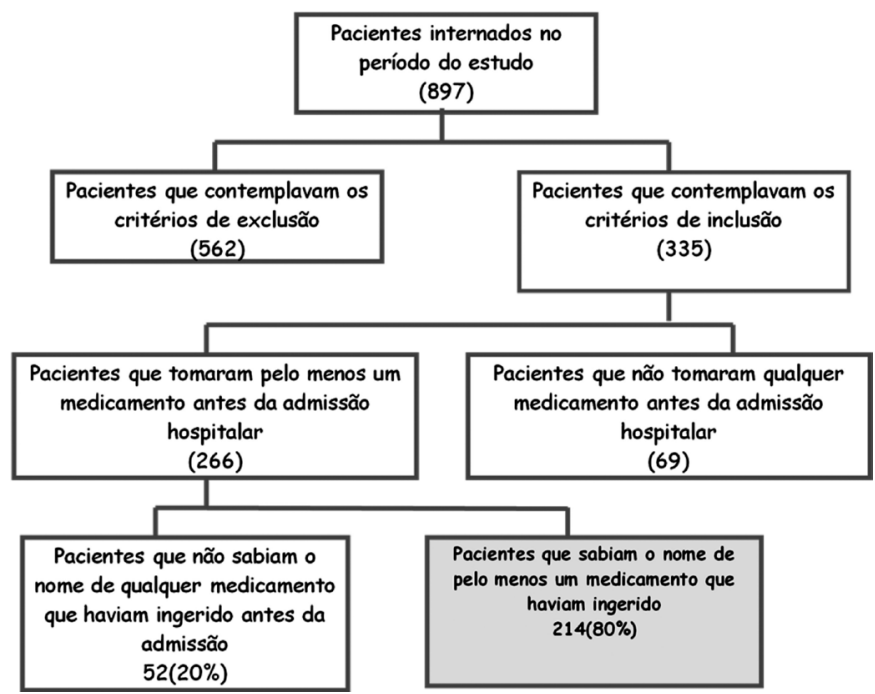

FIGURA 1 - Descrição das informações coletadas durante o estudo, na clínica médica de um Hospital Geral no município de Araraquara - SP. 
Foram identificados 139 pacientes, cujas queixas estavam possivelmente relacionadas com o uso dos medicamentos ingeridos antes da admissão hospitalar. Considerando-se o número total de pacientes internados na clinica médica no período do estudo, estima-se que $15,5 \%$ $(139 / 897)$ destes foram possivelmente internados por RAM. No entanto, considerando-se apenas os pacientes que informaram ter ingerido pelo menos um medicamento antes da internação, praticamente para metade $[52,3 \%$ (139/266)] deles a razão da internação estava possivelmente relacionada a uma RAM.

Quanto ao perfil epidemiológico dos pacientes internados por possíveis RAM, a maioria era composta de idosos [47,5\%(66/139)] e mulheres [62,5\%(87/139)], no entanto, não houve diferença estatisticamente significativa entre gênero, faixa etária e admissão hospitalar relacionada ao uso do medicamento (Tabela I).
Os 214 pacientes que lembravam os nomes dos medicamentos ingeridos antes da internação relataram o uso de 455 medicamentos sob prescrição, 140 medicamentos isentos de prescrição e 114 medicamentos sujeitos a controle especial e, em $73,6 \%, 68,8 \%$ e $90 \%$, respectivamente, a RAM descrita na literatura estava associada com a razão da internação. Quanto à essencialidade dos medicamentos, $46,2 \%$ faziam parte da lista RENAME.

Os pacientes relataram o uso de 218 diferentes fármacos. Destes, os dez fármacos mais freqüentes, cujas RAM estavam possivelmente relacionadas com as razões das admissões hospitalares foram os antihipertensivos e cardiovasculares (4), antitérmicos e antipiréticos (3), isentos de prescrição médica, antiulceroso (omeprazol), hipolipemiante (sinvastatina) e antiasmático (formoterol) (Tabela II). Os pacientes tinham ingerido, em média, 3,6 medicamentos antes da admissão. Quanto maior o número

TABELA I - Freqüência das possíveis reações adversas a medicamentos (RAM) como causa das admissões hospitalares, segundo a faixa etária e gênero

\begin{tabular}{lccccc}
\hline \multirow{2}{*}{ Faixa etária (anos) } & \multicolumn{2}{c}{ RAM identificada } & \multicolumn{2}{c}{ RAM não identificada } & \multirow{2}{*}{ Total N (\%) } \\
\cline { 2 - 5 } & Homens (\%) & Mulher (\%) & Homens (\%) & Mulher (\%) & \\
\hline $18-40$ & 1,1 & 7,5 & 4,9 & 10,9 & $65(24,5)$ \\
$41-60$ & 7,9 & 10,9 & 6,0 & 9,4 & $91(34,2)$ \\
$>60$ & 10,5 & 14,3 & 6,4 & 10,1 & $110(41,3)$ \\
\hline Total & 19,5 & 32,7 & 17,4 & 30,4 & $266(100)$ \\
\hline
\end{tabular}

TABELA II - Os dez fármacos mais freqüentes, cujas reações adversas (RAM) estavam possivelmente relacionadas com à razão da admissão hospitalar

\begin{tabular}{|c|c|c|}
\hline Fármacos & RAM $(\mathrm{N})$ & Principais Sintomas \\
\hline omeprazol & 16 & $\begin{array}{l}\text { Broncoespasmo, fraqueza muscular, dores musculares, vômito, distúrbios gastrintestinais, } \\
\text { pneumonia. }\end{array}$ \\
\hline dipirona & 12 & $\begin{array}{l}\text { Insuficiência renal, ataque asmático, dispnéia, broncoespasmo grave, inflamação na } \\
\text { mucosa, dor de cabeça. }\end{array}$ \\
\hline ácido acetilsalicílico & 11 & Tontura, broncoespasmo, distúrbios gastrintestinais, osteoartrite, dispnéia, ICC**. \\
\hline captopril & 10 & $\begin{array}{l}\text { Alteração da PA*, alteração dos batimentos cardíacos, infarto do miocárdio, ICC**, } \\
\text { broncoespasmo. }\end{array}$ \\
\hline atenolol & 8 & Sonolência, alteração da PA, náusea, ICC*. \\
\hline paracetamol & 8 & Asma, retenção urinária, febre, pneumonia, alterações do batimento cardíaco. \\
\hline amiodarona & 7 & Náusea, vômito, pneumonia, rash cutâneo, alterações no batimento cardíaco. \\
\hline sinvastatina & 7 & $\begin{array}{l}\text { Distúrbios gastrintestinais, tontura, vertigem, diabetes medicamentosa, pneumonia, } \\
\text { dispnéia. }\end{array}$ \\
\hline enalapril & 6 & Alteração da PA*, dor de cabeça. \\
\hline formoterol & 6 & Exacerbação da asma, broncoespasmo. \\
\hline
\end{tabular}

*PA- pressão arterial / ** ICC- insuficiência cardíaca congestiva 
de medicamentos ingeridos antes da admissão hospitalar, maior a possibilidade destes estarem relacionados com a admissão hospitalar (Tabela III). Identificou-se correlação positiva entre o número de medicamentos ingeridos e a possibilidade de ocorrência das RAM ( $\mathrm{r}=0,77028$, $\mathrm{p}<0,05$, IC95\%).
As queixas mais comuns que levaram os pacientes a procurar o hospital, relacionadas possivelmente a RAM foram: diarréia, náuseas, alteração da pressão arterial, hipo ou hiperglicemia, asma, tonturas, dores de cabeça (Tabela IV).

TABELA III - Freqüência do número de medicamentos ingeridos pelos pacientes antes da admissão hospitalar (AH) e o número de pacientes com possíveis reações adversas a medicamentos (RAM) relacionadas com a admissão hospitalar

\begin{tabular}{cccc}
\hline $\begin{array}{c}\text { Medicamentos } \\
\text { ingeridos (N) }\end{array}$ & $\begin{array}{c}\text { Pacientes } \\
\text { Entrevistados }(\mathrm{N})\end{array}$ & $\begin{array}{c}\text { Pacientes com } \\
\left.\text { RAM x AH* }^{*}\right)\end{array}$ & $\%$ \\
\hline 1 & 40 & 17 & 42,5 \\
2 & 49 & 28 & 57,1 \\
3 & 37 & 24 & 64,8 \\
4 & 29 & 22 & 75,8 \\
5 & 20 & 14 & 70 \\
6 & 12 & 10 & 83,3 \\
7 & 12 & 10 & 83,3 \\
8 & 6 & 5 & 83,3 \\
9 & 6 & 6 & 100 \\
10 & 1 & 1 & 100 \\
11 & 1 & 1 & 100 \\
23 & 1 & 1 & 100 \\
\hline TOTAL & 214 & 139 & 64,9 \\
\hline
\end{tabular}

*correlação positiva; $\mathrm{R}=0,77028, \mathrm{p}<0,05$ (IC95\%)

TABELA IV - Principais sintomas (por sistemas) relatados pelos pacientes na admissão hospitalar que, possivelmente, estavam relacionados com uma reação adversa a medicamento (RAM) utilizado antes da internação

\begin{tabular}{lccl}
\hline SISTEMAS & N & \% Acumulada & PRINCIPAIS SINTOMAS (freqüência) \\
\hline Digestório & 80 & 20,5 & Distúrbios gastrintestinais (20), dor abdominal (17), náuseas (11), vômitos (9) \\
Respiratório & 79 & 40,7 & Broncoespasmos (28), asma (12), dispnéia (10), pneumonia (5) \\
Circulatório & 71 & 58,9 & $\begin{array}{l}\text { Alteração da PA* (23), alteração no batimento cardíaco (11), infarto do miocárdio } \\
\text { (6), ICC** (5) }\end{array}$ \\
SNC & 54 & 72,8 & Tontura (17), sonolência (8), dor de cabeça (7), vertigem (5) \\
Endócrino & 21 & 78,2 & Diabete medicamentosa (14), resistência à insulina (3) \\
Geniturinário & 20 & 83,3 & Insuficiência renal (7), retenção urinária (5), nefrotoxicidade (3) \\
Hematopoiético & 10 & 85,9 & Trombocitopenia (2), trombose (2), agranulocitose (1) \\
Osteomuscular & 9 & 88,2 & Dores musculares (6), osteoartrite (1), inflamações articulares (1) \\
Dermatológico & 8 & 90,3 & Inflamação (4), prurido (2), rash cutâneo (1), escoriações (1) \\
Gravidez & 5 & 91,5 & Contrações precoces (4) \\
Oftalmológico & 1 & 91,8 & Distúrbios visuais (1) \\
Não específico & 32 & 100 & Fraqueza (12), tremedeira (4) sintomas gripais (3), febre (3) \\
Total & 390 & 100 &
\end{tabular}

*PA- pressão arterial / ** ICC- insuficiência cardíaca congestiva 


\section{DISCUSSÃO}

No hospital objeto do estudo observou-se que 15,5\% de todas as internações da clinica médica ocorreram, possivelmente, pelo uso de medicamentos. Beijer et al. (2002) ao avaliarem 68 estudos, observaram que a proporção de internação hospitalar por RAM varia de $2,4 \%$ a $35,1 \%$ para a população não idosa e de $6,6 \%$ a $41,3 \%$ para a população idosa. Zargarzadeh et al. (2007) identificaram que 11,5\% das internações eram por problemas relacionados ao medicamento, sendo que $81 \%$ dos problemas eram ineficácia terapêutica e apenas 19\% eram RAM. Os autores reconhecem que $92 \%$ destas internações poderiam ser prevenidas. Hallas et al. (1990) afirmam que a não adesão ao tratamento é um fator significativo para admissão hospitalar, pois um terço dos pacientes foram internados por sintomas de não adesão ao tratamento. No presente estudo, estima-se que dois terços das admissões poderiam ter sido por não adesão ao tratamento e/ou ineficácia terapêutica.

As admissões hospitalares por problemas relacionados a medicamentos foram mais freqüentes em idosos e mulheres. No entanto, não se observaram diferenças estatisticamente significativas nos dados do presente estudo. Caamaño et al. (2005) e Pouyanne et al. (2006) afirmam que as admissões por RAM são mais freqüentes em idosos e mulheres. Em uma revisão sistemática de estudos descritivos, observou-se que os idosos são quatro vezes mais hospitalizados por RAM do que os não idosos (Beijer et al., 2002), mesmo com a tendência das RAM serem menos reconhecidas nos pacientes idosos (Mannesse a, b et al., 2000).

Onder et al. (2002) afirmam que os fatores que predispõem a admissão hospitalar por RAM são, principalmente, a idade e o número de medicamentos ingeridos. Os dados do presente estudo também demonstram uma relação direta entre o número de medicamentos ingeridos e possível internação por RAM.

Quanto aos critérios de essencialidade dos medicamentos, ou seja, pertencerem à Relação Nacional de Medicamentos Essenciais (RENAME**), cujos critérios para seleção são eficácia, qualidade, segurança e preço baixo, não significa que estes estão isentos de problemas relacionados ao seu uso, tanto que $46,5 \%$ dos medicamentos relacionados com a internação eram classificados como essenciais. Portanto, a seleção de medicamentos com características essenciais é apenas um dos aspectos para a promoção do uso racional de medicamentos (Secretaria de Políticas de Saúde, 2000), sendo imprescindíveis também a prescrição, a dispensação e o acompanhamento farmacoterapêutico. Pirmohamed et al. (2004) ressaltam que os fármacos mais antigos e conhecidos estão mais comumente relacionados com as causas de admissão.

Os medicamentos mais freqüentemente utilizados $(64,2 \%)$ antes da admissão foram os medicamentos sob prescrição médica, principalmente os de uso crônico. No entanto, nem todos foram adquiridos mediante a apresentação dos receituários próprios, o que pode caracterizar uma farmacoterapia sem o acompanhamento adequado do médico, ou seja, um uso irracional de medicamento, cujas internações poderiam ser evitadas caso houvesse acompanhamento adequado durante o uso. A maioria das internações por RAM pode ser prevenida, uma vez que se conheça o mecanismo de ação dos fármacos. Geralmente, elas refletem uma prescrição inadequada, monitoramento inadequado ou simplesmente negligência no uso (Onder et al., 2002).

Apesar de menos freqüente (16,1\%), o relato do uso de medicamentos sujeitos ao controle especial* antes da admissão hospitalar, quase sempre $(90 \%)$, era o motivo da internação e estava relacionado com RAM relatada na literatura. Dados corroboram a necessidade de um controle mais rigoroso das prescrições e da dispensação destes medicamentos, pois as conseqüências de seu uso irracional podem levar à dependência e a outros problemas sociais (Noto et al., 2002).

Os medicamentos isentos de prescrição $(19,7 \%)$ também tiveram sua participação nas admissões hospitalares, sendo que três dos dez fármacos mais freqüentes foram o ácido acetilsalicílico, dipirona e paracetamol. Os resultados ratificam a necessidade de orientação de uso destes medicamentos, principalmente nas farmácias e drogarias, pois os mesmos são isentos de prescrição, mas não são isentos de orientação farmacêutica sobre a posologia, advertências, precauções e identificação de possíveis interações medicamentosas quando utilizados concomitantemente. Os dados sugerem a necessidade da participação efetiva dos profissionais farmacêuticos responsáveis por farmácias e drogarias, na promoção do uso racional de medicamentos, promovendo orientação aos usuários, principalmente daqueles que fazem uso contínuo de medicamentos, os que se automedicam e os polimedicados.

Beijer e Blaey (2002) identificaram vários estudos sugerindo que o acompanhamento do uso de medicamentos pelos farmacêuticos, principalmente para pacientes idosos, pode prevenir grande parte do complexo problema das RAM relacionadas à admissão hospitalar. As sugestões vão ao encontro dos resultados deste estudo, uma vez que a maioria dos internados era de pacientes idosos que faziam uso de medicamentos crônicos (captopril, atenolol, enalapril, sinvastatina, formoterol, entre outros) e os seus 
sintomas poderiam ser evitados se conhecido o mecanismo de ação. Estima-se que entre $67 \%$ e $75 \%$ dos casos podem ser prevenidos e que são negligenciados (Howard et al., 2003; Onder et al., 2002).

As complicações geralmente observadas em estudos anteriores foram sintomas do trato gastrintestinal (Caamaño et al., 2005; Mannesse a, b et al., 2000; Onder et al., 2002; Pouyanne et al., 2000), hemorragias (Onder et al., 2002; Pouyanne et al., 2000), complicações metabólicas (Caamaño et al., 2005; Onder et al., 2002) e síncopes (Caamaño et al., 2005). Os sintomas mais freqüentes neste estudo foram complicações do trato-gastrintestinal $(14,6 \%)$, broncoespasmos $(7,2 \%)$, alteração da pressão arterial $(5,9 \%)$, diabetes medicamentosa $(4,3 \%)$ e tontura $(4,3 \%)$.

Diante dos resultados deste estudo, mesmo que preliminares, algumas medidas de prevenção poderiam ser propostas, tais como, melhorar a adesão ao tratamento medicamentoso pelos pacientes(Hallas et al., 1990), melhor julgamento na escolha dos medicamentos pelos médicos (Beijer et al., 2002; Classen et al., 1997), melhorar a comunicação entre os pacientes e os profissionais da saúde (Beijer et al., 2002), diminuindo, desta forma, gastos desnecessários com internação e tempo de internação(Classen et al., 1997) e, conseqüentemente, evitando complicações maiores como os óbitos em hospitais pelo uso inadequado do medicamento (Juntti-Patinen, 2002).

\section{CONCLUSÕES}

- $\quad$ Estima-se que $15,5 \%$ das internações ocorreram devido a RAM, sendo que dois terços destas podem ter sido relacionados à ineficácia terapêutica ou a não adesão ao tratamento;

- As admissões hospitalares por RAM são mais freqüentes em idosos e mulheres, no entanto, neste estudo não houve diferenças estatisticamente significativas;

- Quanto maior o número de medicamentos ingeridos antes da internação, maior a probabilidade da causa da admissão hospitalar ter ocorrido em função de uma RAM;

- Os fármacos cujas RAM estavam mais freqüentemente relacionadas com a internação, foram: omeprazol, dipirona, ácido acetilsalicílico, captopril, atenolol, paracetamol, amiodarona, sinvastatina, enalapril e formoterol; e os sintomas mais freqüentes são complicações do trato gastrintestinal $(14,6 \%)$, broncoespasmos $(7,2 \%)$, alteração da pressão arterial $(5,9 \%)$, diabetes medicamentosa $(4,3 \%)$ e tontura $(4,3 \%)$.

\section{AGRADECIMENTOS}

Ao Programa de Apoio ao Desenvolvimento Científico da Faculdade de Ciências Farmacêuticas da Universidade Estadual Paulista (PADC-FCF/UNESP) pelo apoio financeiro e institucional e ao Departamento de Psicobiologia da Universidade Federal de São Paulo (UNIFESP) pelo apoio institucional.

\section{REFERÊNCIAS}

BEIJER, H.J.M.; BLAEY, C.J. Hospitalizations caused by adverse drug reactions (ADR): a meta-analysis of observational studies. Pharm. World Sci., v.24, p.46-84, 2002.

BRASIL. Portaria SVS/MS n. ${ }^{\circ}$ 344, 12 de maio de 1998. Diário oficial da República Federativa do Brasil, Brasília, 19 maio 1998. Disponível em: <http://e-legis.anvisa.gov.br/leisref/ public/showAct.php?id=17235\&word=\#'>. Acesso em: 25 Set. 2008.

BRASIL. Portaria GM/MS n ${ }^{\circ} 2.475$, de 13 de outubro de 2006. Diário Oficial da União. Brasília, 18 de outubro de 2006. Disponível em: http://74.125.47.132/ search?q=cache:xQoq5h89ab0J:ftp://ftp.saude. sp.gov.br/ftpsessp/bibliote/informe_eletronico/2007/

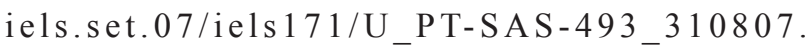
$\mathrm{pdf}+$ Portaria $+\mathrm{GM} / \mathrm{MS}+\mathrm{n} \% \mathrm{C} 2 \% \mathrm{BA}+2.475 \& \mathrm{hl}=\mathrm{pt}-$ $\mathrm{BR} \& \mathrm{ct}=\mathrm{clnk} \& \mathrm{~cd}=1 \& \mathrm{gl}=$ br. Acesso em: 25 Set. 2008.

CAAMAÑO, F.; PEDONE, S.; ZUCCALA, C.P. SocioDemografic factors related to the prevalence of adverse drug reaction at hospital admission in an elderly population. Arch. Gerontol. Geriatr., v.40, p.45-52, 2005.

CLASSEN, D.C.; PESTOTNIK, S.L.; EVANS, R.S.; LLOYD, J.F.; BURKE, J.P. Adverse Drug Events in hospitalized Patients. JAMA, v.277, p.301-306, 1997.

GREEN, C.F.; MOTTRAM, D.R.; ROWE, P.H.; PIMOHAMED M.. Adverse drug reactions as a cause of admissions to an acute medical assessment unit: a pilot study. J. Clin. Pharm. Ther., v.25, p.355-361, 2000.

HALLAS, J.; HARVALDE, B.; GRAM, L.F.; GRODUM, E.; BROSEN, K.; HAGHEFELT, T.; DAMSBO, N.. Drug related hospital admissions: the role of definitions and intensity of data collection, and the possibility of prevention. J. Intern. Med., v.228, p.83-90, 1990. 
HOWARD, R.L.; AVERY A.J.; HOWARD, P.D.; PARTRIDGE, M.. Investigation into the reasons for preventable drug related admissions to a medical admissions unit: observational study. Qual. Saf. Health Care, v.12, p.280-285, 2003.

JUNTTI-PATINEN, L.; NEUVONEM, P.J. Drug-related deaths in a university central hospital. Eur. J. Clin. Pharmacol., v.58, p.479-482, 2002.

MANNESSE, C.K.; DERIKX, F.H.M.; RIDEER, M.A.J.; VELDE, M.; VAN DER CARMMEN, T.J.M.. Contribution of adverse drug reactions to hospital admission of older patients. Age Ageing, v.29, p.35-39, 2000a.

MANNESSE, C.K DERIKX, F.H.M.; RIDEER, M.A.J.; VELDE, M.; VAN DER CARMMEN, T.J.M.. Contribution of adverse drug reactions to hospital admission of older patients. Age Ageing, v.29, p.79-81, 2000 b.

MINISTÉRIO DA SAÚDE. Classificação estatística internacional de doenças e problemas relacionados à saúde - CID-10. Brasília; MS; 1998. Disponível em: <URL: http:// www.cid10.hpg.ig.com.br/>. Acesso em: 28 Fev. 2007.

NOTO, A.R.; CARLINI, E.A.; MASTROIANNI, P.C.; ALVES, V.C.; GALDURÓZ, J.C.F.; KUROIWA, W. et al. Análise da prescrição e dispensação de medicamentos psicotrópicos em dois municípios do Estado de São Paulo. Rev. Bras. Psiquiatr., São Paulo, v. 24, n. 2,2002 . Disponível em: <http://www. scielo.br/scielo.php?script $=$ sci_arttext\&pid $=\mathrm{S} 1516$ $44462002000200006 \& \operatorname{lng}=p t \& n r m=i s o>$. Acesso em: 10 Maio 2008.

ONDER, G.; PEDONE, C.; LANDI, F.; CESARI, M.; VEDOVA, C.D.; BERNABEI, R.; GAMBASSI, G.. Adverse drug reactions as cause of hospital admissions: results from the Italian group of pharmacoepidemiolgogy in the elderly (GIFA). JAGS, v.50, p.1962-1968, 2002.

PEREIRA, M.G.. Métodos empregados em epidemiologia. In: PEREIRA, M.G. Epidemiologia: teoria e prática. 1. ed. Rio de Janeiro. Guanabara: 1995. p.269- 288.
PIRMOHAMED, M.; JAMES, S.; MEAKIN, S.; GREEN, C.; SCOTT, A.K.; WALLEY, T.J.; FARRAR, K., PARK, B.K.; BRECKENRINDGE, A.M.. Adverse drug reactions as cause of admission to hospital: prospective analysis of 18.820 patients. BMJ, v.329, p.15-19, 2004.

PFAFFENBACH, G.; CARVALHO, O.M.; BERGSTENMENDES, G.. Reações adversas a medicamentos como determinantes da admissão hospitalar. Rev. Assoc. Med. Bras., v.48, p.237-241, 2002.

POUYANNE, P.; HARAMBURU, R.; IMBS, J.L.; BEGAUD, B. Admissions to hospital caused by adverse drug reactions: cross sectional incidence study. BMJ, v.320, p.1036, 2000 .

SCHNEEWEISS, S.; GÖTTLER, M.; HASFORD, J.; SWOBADA, W.; HIPPIUS, M.; HOFFMANN, A.K.; RIETHING, A.K.; KRAPPWEIS, J.. First results from an intensified monitoring system to estimate drug related hospital admissions. Br. J. Clin. Pharmacol., v.52, p.196-200, 2001.

SECRETARIA DE POLÍTICAS DE SAÚDE. Política Nacional de Medicamentos. Rev. Saúde Pública, v.24, p.206-209, 2000 .

WHO. International drug monitoring: the role of the national centers. WHO Technical Report Series n. 498. Genebra: WHO, 1972. 47p.

WHO. World Health Organization Collaborating Centre for Drug Statistics Methodology. ATC/DDD Index 2008. Available at: $<$ http://www.whocc.no/atcddd $>$. Access on: Feb. 07th. 2008 .

ZARGARZADEH, A.H.; EMANI, M.H.; HOSSEINI, F. Drugrelated hospital admissions in a generic pharmaceutical system. Clin. Exp. Pharmacol. Physiol., v.34, p.494-498, 2007.

Recebido para publicação em 14 de maio de 2008. Aceito para publicação em 07 de agosto de 2008. 